Environnement Urbain

Urban Environment

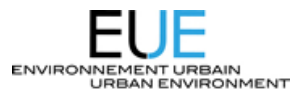

\title{
Les inondations de 1733 et 1740 à Grenoble : L'évolution de la résilience dans l'après catastrophe
}

\section{Théo Blanchard}

Volume 2, 2008

Inondations en milieux urbains et périurbains

Floods in urban and suburban areas

URI : https://id.erudit.org/iderudit/019222ar

DOI : https://doi.org/10.7202/019222ar

Aller au sommaire du numéro

\section{Éditeur(s)}

Institut national de la recherche scientifique

ISSN

1916-4645 (numérique)

Découvrir la revue

\section{Citer cet article}

Blanchard, T. (2008). Les inondations de 1733 et 1740 à Grenoble : L'évolution de la résilience dans l'après catastrophe. Environnement Urbain / Urban

Environment, 2, 73-89. https://doi.org/10.7202/019222ar
Résumé de l'article

En 1733 et en 1740, Grenoble, capitale du Dauphiné, fut frappée par deux des plus graves inondations de l'Isère. La résilience socio-économique de ce territoire urbain et périurbain met en évidence l'importance des arbitrages des différents acteurs dans la capacité de survie du système grenoblois au-delà de la catastrophe.

La résilience sera considérée comme un processus de retour du système touché par une inondation vers la «normale ». La conservation et l'adaptation de la société résultent d'un ensemble de phénomènes dûs à des tensions ou, au contraire, à la cohésion, qui mettent en exergue des dynamiques sociales et territoriales. La catastrophe réveille une sensibilité accrue aux vulnérabilités du système. Elle participe ainsi à l'élaboration d'une construction plus large du risque d'inondation au XVIII ${ }^{\mathrm{e}}$ siècle. 


\title{
LES INONDATIONS DE I 733 ET I 740 À GRENOBLE : L'ÉVOLUTION DE LA RÉSILIENCE DANS L'APRÈS CATASTROPHE
}

\author{
Théo BLANCHARD
}

\begin{abstract}
I RÉSUMÉ
En 1733 et en 1740, Grenoble, capitale du Dauphiné, fut frappée par deux des plus graves inondations de I'Isère. La résilience socio-économique de ce territoire urbain et périurbain met en évidence l'importance des arbitrages des différents acteurs dans la capacité de survie du système grenoblois au-delà de la catastrophe.

La résilience sera considérée comme un processus de retour du système touché par une inondation vers la «normale ». La conservation et l'adaptation de la société résultent d'un ensemble de phénomènes dûs à des tensions ou, au contraire, à la cohésion, qui mettent en exergue des dynamiques sociales et territoriales. La catastrophe réveille une sensibilité accrue aux vulnérabilités du système. Elle participe ainsi à l'élaboration d'une construction plus large du risque d'inondation au XVIIII siècle.
\end{abstract}

MOTS-CLÉS - résilience, inondation, Grenoble, $18^{\mathrm{e}}$ siècle, construction du risque

\section{- ABSTRACT}

Grenoble, the capital city of Dauphiné, was flooded twice by the Isère River, once in 1733 and a second time in 1740. Socio-economic resilience of this urban and suburban district points out how arbitration by social actors was a key factor in the ability to survive of the Grenoblian system after the disaster.

The study of resilience, considered as the "back-to-normal" process of the impacted system, shows social and territorial dynamics, tensions and cohesions at work in order to preserve and adapt the society. Catastrophe also awakens a greater sensitivity to the system's vulnerable spots, building a growing awareness of flood risk during the 18 th century.

KEYWORDS - resilience, flood, Grenoble, $18^{\text {th }}$ century, risk construction 


\section{INTRODUCTION}

Au XVIII ${ }^{\text {e }}$ siècle, la catastrophe apparaît comme un «miroir révélateur des fragilités internes de l'édifice urbain et du territoire " (Cœur, 2003). L'occurrence d'une inondation majeure exerce un effet brutal et déstabilisateur sur la société urbaine touchée. La dynamique enclenchée par chaque événement se prolonge sur le terrain durant des jours, voire des années après l'inondation. En effet, la catastrophe représente une menace grave pour les sociétés, à la fois dans leur intégrité à court terme et dans leurs structures et leur fonctionnement à long terme (Berlioz et Quénet, 2000). Néanmoins, les sociétés structurées du $X V I I^{e}$ siècle savaient dépasser les pièges entropiques' pour s'engager dans un processus de résilience.

La notion de « résilience » est encore soumise à des débats. Issue du monde de la physique et reprise par l'écologie, elle est introduite en sciences sociales par le biais de la systémique. La résilience se définit, dans ce contexte, comme la capacité d'un système social à absorber une perturbation et à se réorganiser, alors qu'il subit des changements, de façon à retrouver essentiellement ses mêmes fonctions, sa structure, son identité et ses réactions (Walker et al., 2004). La société touchée par la perturbation doit survivre audelà de la catastrophe, en conservant un niveau acceptable de structure et de fonctionnement « traditionnel $»^{2}$.

La résilience a pu être considérée comme une « composante de la vulnérabilité » (Pelling, 2003), puisque ces deux concepts s'intéressent aux mêmes facteurs (sociaux, économiques, politiques, culturels, etc.). Malgré tout, la résilience se distingue de la vulnérabilité $^{3}$ car elle donne un caractère actif à la société confrontée au risque. La résilience envisage la capacité des sociétés à gérer les crises et les catastrophes et la manière dont elles le font (Gaillard, 2007). Cette «digestion » des impacts des aléas naturels par les sociétés varie dans l'espace et dans le temps. La résilience n'est donc pas seulement une capacité de cicatrisation des plaies. Elle apparaît bien comme un processus qui entraîne la société concernée

\footnotetext{
I Entropie: force qui conduit spontanément la matière vers le désordre.

${ }^{2}$ La notion de résilience est également utilisée en psychologie. Selon Boris Cyrulnik, il s'agit de la capacité d'un individu, touché par un traumatisme, à surmonter le handicap physique ou social qui en résulte pour « vivre avec » (Cyrulnik, 200I).

3 La vulnérabilité ne prend en compte que la propension des individus à souffrir des dommages et à transformer l'occurrence d'un aléa naturel en catastrophe (Gaillard, 2007).
}

vers son rétablissement. Les dynamiques impulsées par chaque acteur conditionnent le rythme et l'orientation de ce processus vers une adaptation ou une transformation de la société (Walker et al., 2004) ${ }^{4}$.

Le concept de résilience a été amplement étudié en situation de catastrophe, en ce qui concerne les sociétés industrielles contemporaines et, plus récemment, les sociétés traditionnelles contemporaines (Gaillard, 2007). Les travaux montrent la très grande diversité des formes et des aboutissements de la résilience pour les différentes sociétés. C'est pourquoi cette notion est difficile à généraliser et à modéliser. L'introduire dans l'histoire sociale du risque nécessite de prendre en compte le contexte et les spécificités de la structure et du fonctionnement habituel des sociétés préindustrielles concernées ${ }^{5}$. L'historien du risque doit s'accommoder des lacunes dans les sources disponibles et se garder de toute surinterprétation conceptuelle. La perspective historique permet néanmoins d'appréhender l'ensemble du processus de résilience dans sa complexité. En effet, le temps de la résilience socio-économique, replacé sur la longue durée, fait apparaître les liens étroits entre la société et les risques qui l'environnent.

L'examen empirique des résonances des inondations catastrophiques de 1733 et 1740, à Grenoble, permettra de faire ressortir certaines caractéristiques de la résilience de cette ville. Ce sera également l'occasion de mettre en lumière les incidences de ce processus sur le rapport aux risques d'une société urbaine et périurbaine du $\mathrm{XVIII}{ }^{\mathrm{e}}$ siècle. Cet article part de l'hypothèse que la société grenobloise était résiliente, afin se focaliser sur l'étude du processus. L'évolution de la résilience est un prisme d'étude qui met en exergue certaines caractéristiques des sociétés urbaines dans le contexte où une catastrophe s'est produite. Les étapes de la résilience économique ne sont pas suffisantes pour appréhender la complexité du processus social et culturel. Nous distinguerons donc deux phases majeures dans la résilience sociale. Le premier temps de la résilience paraît court. Une urgence sociale active vise à maintenir autant que possible la cohésion du système touché. A plus long terme, l'approfondissement du processus de résilience conduit la société vers le retour à la normale. Au cours

\footnotetext{
${ }^{4}$ Il s'agit donc à la fois d'approcher la réponse des sociétés et des individus à la catastrophe.

${ }^{5}$ En ce sens les aspects de la résilience définis par B. Walker peuvent apporter des clefs de compréhension: la latitude, la résistance, la précarité et la panarchie (Walker et al., 2004). Selon cette approche l'analyse doit avoir une perspective multi-scalaire.
} 
de cette période, la notion de conscience du risque s'exacerbe. Cela participe d'une prise de conscience du risque au sein de la société. Enfin les conséquences de l'inondation engendrent des mutations dans la gestion durable du territoire à risque de Grenoble.

\section{GRENOBLE INONDÉ, UNE CATASTROPHE RÉITÉRÉE}

« Grenoble et sa campagne, c'est comme la haute mer » (Blanc, I733, v. I5I). Cette situation incongrue, exceptionnelle et catastrophique frappe la ville deux fois de façon rapprochée, en 1733 et 1740 . II n'en fallait pas moins pour faire entrer ces deux inondations de l'Isère parmi les désastres majeurs de l'histoire de Grenoble.

\section{I.I Grenoble et sa campagne : une cité, un territoire, un système}

Si modeste soit cette cité de 22600 habitants, blottie au cœur des Alpes, Grenoble n'en demeure pas moins une ville importante dans le royaume de France (Favier, 1993). Capitale du Dauphiné, province parlementaire et apanage de l'héritier du trône, Grenoble concentre les enjeux politiques. Place fortifiée, elle assure la protection militaire du royaume face aux ambitions savoyardes et elle sert d'étape aux expéditions françaises en Italie. Siège d'un évêché, la ville abrite de nombreuses communautés monastiques. Nœud de communication aux débouchés des grandes vallées alpines, la cité abrite de nombreux commerçants. Enfin, seule ville au cœur d'un espace rural et montagnard, Grenoble polarise un important territoire et elle prospère grâce à cette influence. II s'agit donc de conserver « une ville capitale de province et tous ses environs $\|^{6}$. Les enjeux sont de taille.

Cette étude se concentrera sur la ville de Grenoble et ses faubourgs. La petite plaine inondable qui l'entoure sera incluse dans le territoire urbain, puisqu'elle est en interaction étroite avec la ville. En effet, les terres qui l'environnent sont possédées par les notables et les monastères urbains. Elles sont confiées en fermage ${ }^{6}$ aux habitants des faubourgs et des hameaux, qui assurent alimentation et revenus à la cité. II ne faut donc pas considérer le système urbain grenoblois comme enclos dans ses remparts, mais plutôt de l'étendre au territoire polarisé par la ville-centre et englobant son espace " périurbain». Au cœur de la catastrophe, ces deux espaces ne subiront pas de la même façon les impacts des inondations. Pourtant tout au long de la résilience, leur destin sera commun.

La ville est bâtie de part et d'autre de l'Isère, juste en amont de la confluence de celle-ci avec le Drac. Ces deux rivières drainent vers la cluse de Grenoble une grande partie des massifs alpins français. Ce site de plaine fait de Grenoble une ville fréquemment soumise au risque d'inondation (voire figure I).

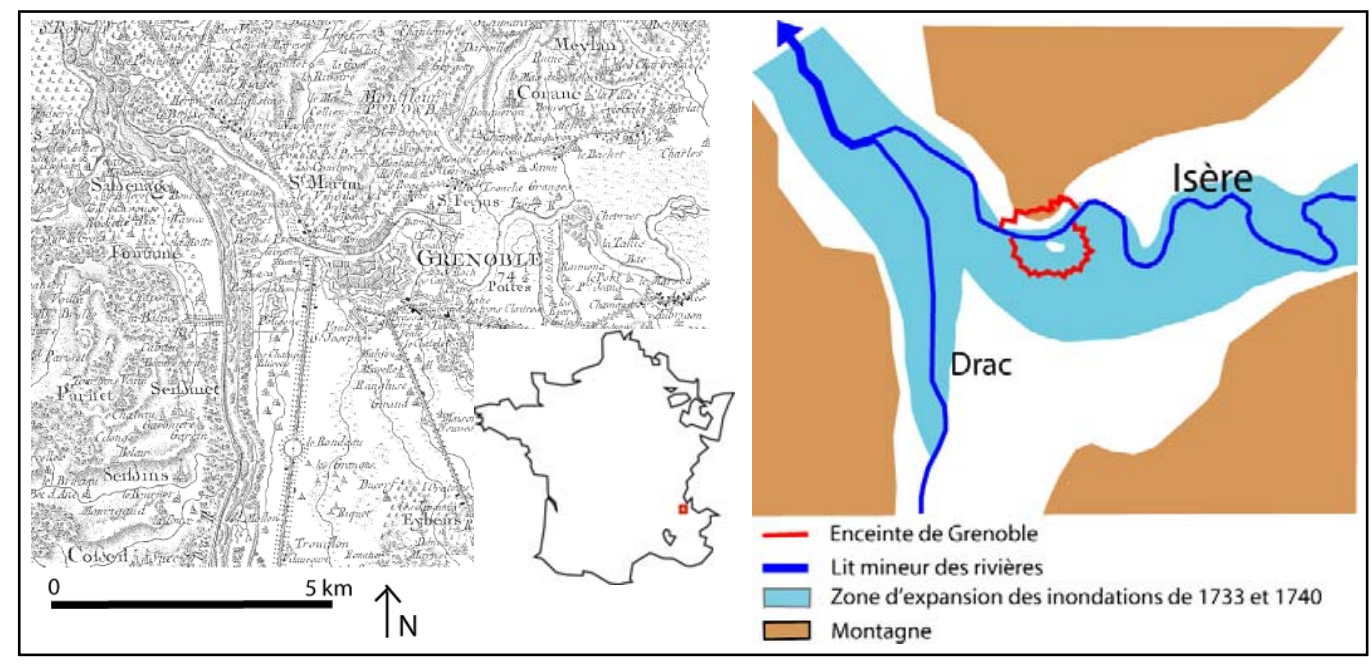

Fig. I - Grenoble et sa plaine inondable

\footnotetext{
${ }^{6}$ La pratique de la mise en fermage par un propriétaire consiste à faire exploiter sa terre par un paysan local en échange d'un loyer annuel. Les obligations et les droits réciproques des deux partis sont stipulés dans le bail à ferme.
} 


\section{I.2 Deux inondations exceptionnelles : 1733 et 1740}

Seize inondations majeures sont recensées dans la région qui nous intéresse du $\mathrm{XVII}{ }^{\mathrm{e}}$ au $\mathrm{XIX}^{\mathrm{e}}$ siècle (Cœur, 2003). La décennie 1730 concentre à elle seule trois de ces événements. Deux des seize inondations affectent durement Grenoble, tandis que trois autres endommagent sa campagne ${ }^{7}$. Cette concentration d'inondations, unique dans l'histoire hydraulique de la ville, est ponctuée par deux paroxysmes: les inondations pluri-centennales de septembre 1733 et de décembre 1740 (Cœur, 2003).

Le I5 septembre 1733, l'Isère aurait atteint une hauteur maximale de 5,57 mètres au-dessus de son niveau moyen, ce qui correspond à un débit de 1900 $\mathrm{m}^{3} / \mathrm{s}$ (Parde, 1928). Ces caractéristiques rarement égalées dans l'histoire de ce cours d'eau justifient l'introduction poignante qu'un notaire grenoblois, Jean-François Marchand, dit le jeune, donne à son récit : « Lundi I 4 septembre I 733 jour de la Croix sur les quatre heures après midi il s'éleva un ouragan des plus furieux qui aye parû depuis bien longtemps qui causa une consternation generalle dans la ville $\Perp^{8}$. Sur l'initiative de l'intendant et du gouverneur militaire de la ville, les portes des enceintes sont fermées et renforcées face à la montée des eaux. Pour les Grenoblois, le répit est de courte durée. Dans la matinée du 15 septembre, les portes cèdent. Le déluge submerge les Grenoblois. L'eau monte jusqu'à 2,60 mètres en ville, mais épargne de rares rues exondées du centre. L'eau stagne durant 24 heures avant de laisser place à « une boüe gluante et puante et en si grande quantité que les rues et places et lieux sont impraticables avec une puanteur extraordinaire ${ }^{9}$. Le mercredi 16 septembre 1733 marque la fin de l'inondation effective et le commencement de l'évacuation progressive des boues. Commence alors la résilience ${ }^{10}$ d'une des plus grandes inondations de l'histoire de Grenoble.

L'histoire se répète pourtant sept ans plus tard, en 1740. «Le 20 décembre sur les cinq heures du soir, l'zère fut elevée de 4 pieds au dessus de ses eaux

\footnotetext{
7 Ces inondations se produisent en octobre 1732, du 14 au 16 septembre 1733, en juin 1737, en octobre et décembre 1739 et du 20 au 24 décembre 1740 .

${ }^{8} \mathrm{AD}$ Isère : $3 \mathrm{E}$ I108/7 : «Relation du déluge arrivé dans Grenoble le 15 septembre 1733 » par J. F. Marchand le Jeune.

${ }^{9}$ Ibid.

10 L'après catastrophe n'est pas seulement le temps des reconstructions, il s'agit également d'enjeux de récupération pour le tissu social.
}

ordinaires " ". Le même scénario semble alors se dessiner: «cette première élévation fit craindre des progrès plus considérables ${ }^{12}$ de l'inondation. Durant la matinée du 21 décembre 1740, les eaux de l'Isère envahissent la ville, à l'exception du même îlot central. Les hauteurs d'eau sont similaires, puisqu'elles ne surpassent que de 15 centimètres celles atteintes lors de l'inondation précédente. La journée du 22 décembre est consacrée à la décrue, mais l'Isère ne rentre définitivement dans son lit que le 24 décembre ${ }^{13}$.

L'impact de ces deux inondations terribles fut durement ressenti par l'ensemble du système grenoblois: les élites urbaines durent se mobiliser " pour nous aider à nous relever plus facilement " (Blanc, 1733, v. 379).

\subsection{Destructions matérielles et paralysie urbaine}

La ville se relève douloureusement. L'inondation «ne la détruisait pas de fond en comble y occasionnerait [néanmoins] de ce ravages affreux. ${ }^{14}$. Aux destructions matérielles s'ajoute l'arrêt, ou à tout le moins le ralentissement des activités quotidiennes. Tout le système économique de Grenoble est mis à mal.

Parmi les destructions, la plus symbolique est la perte d'un pont emporté en 1733, (un autre pont est fortement endommagé à chaque inondation). Les dégradations subies par les portes et pont-levis comptent également parmi les dégâts ayant marqué les esprits. Pourtant, en comparaison de la destruction de certains emblèmes de Grenoble, les pertes subies par les habitants apparaissent comme bien plus conséquentes. L'immobilier urbain des quartiers pauvres est en première ligne. En 1733, «L'lsère passant en rue Saint-Laurent et à la Perrière renversa trois ou quatre maisons ${ }^{15}$. A travers toute la ville 153 autres bâtiments sont en "péril de la chute ${ }^{16}$. En 1740, seules 49 habitations seront répertoriées. Cette

\footnotetext{
" $A D$ Isère : $2 C 816, f^{\circ} 6$ bis : "Mémoire sur le débordement de I'Izère des 21,22 et 23 décembre $1740 \ldots$ ».

12 Ibid.

${ }^{13}$ Ibid.

${ }^{14} A D$ Isère : J $523, f^{\circ} 13$

15 BM Grenoble: R 8742: Témoignage de Pierre Bémond sur l'inondation de 1733 à Grenoble.

${ }^{16}$ AM Grenoble: DD I75: «Copie de la procédure de Messieurs les trésoriers de France au sujet de l'Inondation arrivée à Grenoble les 14 et I5 du même mois. » et AD Isère : $2 \mathrm{C} 816, \mathrm{f}^{\circ} 2$.
} 
vulnérabilité minorée lors de la seconde inondation peut être interprétée comme une conséquence des réparations effectuée suite à la catastrophe de 1733 . Un rapport donne l'estimation des pertes subies par chaque paroissien de Saint-Joseph ${ }^{17}$, faubourg particulièrement sinistré de Grenoble lors de l'inondation de décembre 1740. Au côté de l'immobilier, qui ne représente que $2 \%$ des occurrences de pertes, les dégradations mobilières représentent l'écrasante majorité des pertes déclarées (70\% des occurrences), mais seulement $34 \%$ de la valeur totale.

Les quelques exploitants agricoles de cette paroisse attenante à la ville déclarent $76 \%$ du coût total des destructions ${ }^{18}$. "Dans cette accident presque tous les fermiers ont perdu dans leur ferme les fruits de deux années savoir 1740 et toutes leurs espérances pour $174 I.\rangle^{19}$. Les denrées agricoles perdues et les déficits de récolte estimés comptent chacun pour un quart des destructions de l'inondation. Face à cette carence dans l'approvisionnement direct des marchés urbains, il faudra recourir à l'ouverture des entrepôts royaux et à l'importation de blé pour permettre de satisfaire les besoins alimentaires de la société grenobloise. Cette situation de déficit local est amenée à durer, puisque les terres agricoles (notamment des jardins) sont ravagées. De plus les berges dégradées des cours d'eau sont soumises à une érosion constante. Les fossés sont combles. Les quais de ville et les quelques digues dans la plaine sont renversés en maints endroits.

Ce bref aperçu des dégâts engendrés par l'inondation est incomplet si l'on n'évoque pas la paralysie qui frappe Grenoble aux lendemains des inondations. "Tous les ponts sont détruits et les chemins, défoncés. »(Blanc, I733, v. 348). La boue entrave les déplacements, qui sont si nécessaires dans la période suivant la catastrophe. Le redémarrage des activités économiques n'est donc pas facilité. Le commerce est entravé et l'artisan, démuni de ses outils, doit s'interrompre durant un mois ${ }^{20}$. Par contre, l'activité notariale, annihilée durant l'inondation, est clairement

\footnotetext{
$17 \mathrm{AD}$ Isère : $2 \mathrm{C} 816, \mathrm{f}^{\circ} \mathrm{I}$ : «Observation sur le dénombrement des habitants de la Paroisse de St Joseph qui ont été inondez et sur les pertes qu'ils ont faites. »

18 Les 86 fermiers, laboureurs ou jardiniers ne représentent pourtant que $34 \%$ des 250 foyers de la paroisse.

${ }^{19} \mathrm{AD}$ Isère : $2 \mathrm{C} 816, \mathrm{f}^{\circ} \mathrm{I}$

20 lbid.
}

redynamisée trois jours après chaque décrue ${ }^{21}$. Si les conséquences économiques de l'inondation touchent durement un espace assez large, la durée des effets les plus néfastes ne semble pas être trop longue. Certaines conséquences se font pourtant encore sentir des mois, et même des années, après les destructions. La dimension finale de la catastrophe dépendra alors de la vitesse de rééquilibrage du système (Benassar, 1996). Il faut donc déterminer le rythme et les caractéristiques d'un processus de résilience à dimensions multiples.

\section{DESTRUCTIONS MATÉRIELLES ET PARALYSIE URBAINE}

Des dynamiques internes d'adaptation et de reconstruction du système se conjuguent. Elles portent la société touchée par la catastrophe depuis l'état de «bouleversement », généré par l'aléa naturel, vers celui de «normalité », proche de l'état initial du système. Ce processus comporte différentes phases lors desquelles les priorités et les logiques des acteurs de la société diffèrent. Les dynamiques conjuguées de cohésion et de tensions, qui soutiennent la résilience sociale (voir infra), ne permettent pas de déterminer un rythme spécifique au processus de résilience.

En revanche, les indicateurs de la résilience économique marquent clairement l'existence de phases successives. Le meilleur indicateur de l'activité économique d'une société reste le notaire, qui enregistre quotidiennement les endettements, les locations, les ventes, les devis et les rapports d'expert qui se réalisent chaque jour. Les registres de contrôle d'actes notariés du bureau de Grenoble ${ }^{22}$ recensent chacun de ces contrats. L'étude de ces enregistrements durant les mois qui précèdent et qui suivent chaque inondation permet d'observer de fortes tendances au ralentissement ou à l'accroissement de l'activité économique de la ville d'après les rythmes de contraction de ces actes ${ }^{23}$. Les périodes de forte activité notariale marquent des temps de récupération économique importante après

\footnotetext{
21 L'activité notariale est un bon indicateur du niveau relatif de l'activité économique d'une ville. Celle-ci est quintuplée entre le 18 et le 19 septembre 1733 (AD Isère : 8 C II40).

${ }^{22} \mathrm{AD}$ Isère : $8 \mathrm{C}$ । I40, $8 \mathrm{C}$ । I73 et $8 \mathrm{C}$ । I74

${ }^{23}$ L'interprétation de ce rythme ne doit pas aller au-delà, puisque la formalité des actes notariés ne nous donne pas de renseignement sur les causes de contraction de chacun de ces actes. Nous n'évoquerons pas ici l'analyse détaillée des rythmes de contraction de chaque type d'acte, seul la synthèse qui en découle sera présentée.
} 
la catastrophe ${ }^{24}$. A l'inverse, les périodes de faible activité notariale renverront à des moments de difficulté économique. Les évolutions de cette activité économique accompagnent donc la résilience de l'ensemble du système grenoblois.

Déterminer un aboutissement à ce processus de résilience reste délicat, puisque la société tend vers son état initial sans le recouvrer entièrement. Le rythme de contraction des actes notariés se stabilise au bout de quelques mois à un niveau proche de son état de stabilité avant la catastrophe. Ce retour à l'équilibre marque, selon nous, la fin théorique du processus de résilience économique. La résilience qui fait suite à l'inondation de 1740 paraît bénéficier de l'expérience acquise en 1733, puisqu'elle est moins impulsive et plus rapide que la précédente. En effet, la période de résilience s'étale sur trois mois en 1733 , contre seulement deux mois en $1740^{25}$. Si la durée et l'amplitude de chaque résilience est spécifique, les deux processus sont néanmoins comparables dans leurs rythmes. La résilience économique du système grenoblois face aux inondations similaires de 1733 et I 740 peut donc se schématiser en six phases distinctes (voire figure 2) :

I) Un temps « mort » succède à l'arrivée des eaux en ville et se prolonge au-delà de la décrue: l'activité économique semble paralysée.

2) Au choc succède une phase d'urgence, marquée par un brusque accroissement temporaire de l'activité économique durant dix jours. La forte proportion des endettements dénote un besoin de survie.

3) Après cette première réaction, une phase de stabilisation ponctuée de soubresauts se poursuit durant trois semaines. La faible activité notariale renvoie aux difficultés économiques de la société sinistrée.

4) II s'engage alors une phase de transition entre une déstructuration qui paraît appartenir au passé et une redynamisation de l'économie qui tarde à venir. Il s'agit d'une dépression d'une durée variable dans l'activité notariale, qui reste difficilement explicable.

5) Le dénouement de la résilience économique semble être un redémarrage accéléré des échanges conduisant à une intensité d'activité très forte sur un temps plus long.

6) La résilience économique entière parait intervenir par une décélération puis une réelle stabilisation de l'activité à son niveau moyen de l'avant catastrophe.

L'activité économique grenobloise de la période qui suit la catastrophe fait apparaître successivement : un temps mort et une mobilisation d'urgence, qui peuvent être assimilés à une phase d'urgence ; une stabilisation précaire et une transition difficile, dans lesquelles on peut voir une crise liée aux conséquences de la catastrophe ; un redémarrage accéléré et un retour à la normale, qui marquent une résilience quasi complète du système grenoblois.

Cette chronologie n'est valable que pour le versant

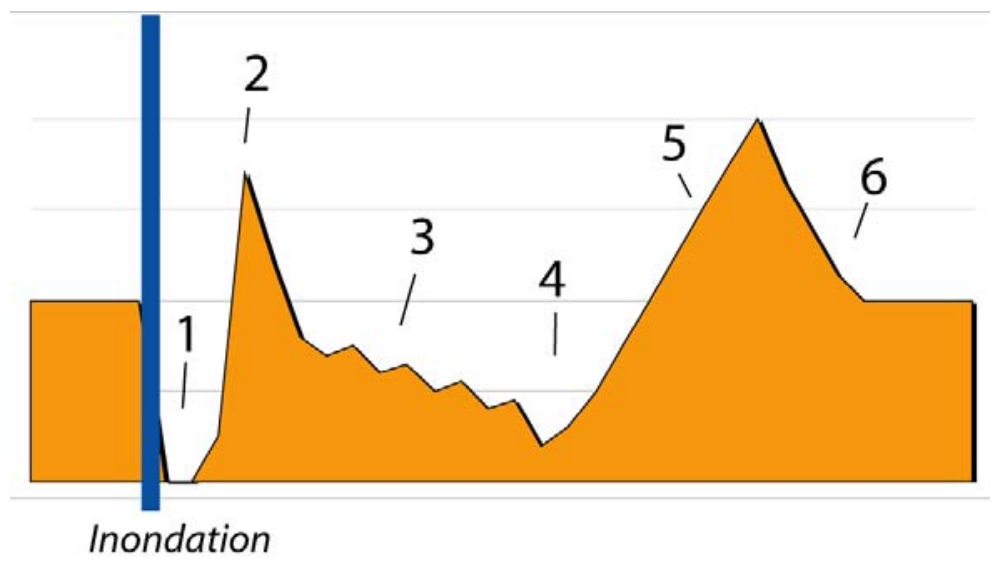

Fig. 2 - Schéma des rythmes de résilience économique de Grenoble (I733 et I740)

\footnotetext{
24 II est possible, avec prudence, d'assimiler ces périodes à des phases de normalisation.

${ }^{25}$ Soit du 15 septembre 1733 jusqu'aux environs du 17 décembre 1733 et du 2I décembre 1740 jusqu'aux environs du 15 février I74I.
} 
économique de la résilience. Les aspects sociaux s'entremêlent et se combinent de façon complexe, laissant apparaître deux dynamiques qui se recoupent dans le temps, mais que nous distinguerons dans cette étude. Une phase d'urgence précède une période de normalisation au cours de laquelle adaptation et transformation (Walker et al., 2004) modèlent le rapport de la société au risque.

\section{LES PREMIERS ÉLANS DE LA RÉSILIENCE : ENTRE SURVIE ET SOLIDARITÉ D'URGENCE}

Dès le déclenchement de l'aléa, au cœur même de la catastrophe, les individus réagissent et s'adaptent en vue d'une meilleure résilience. Ils ne se contentent pas de se protéger. Ils anticipent la survie qui leur sera imposée durant l'inondation et les difficultés qu'ils devront affronter après la décrue. Ces deux aspects peuvent difficilement être dissociés chez les acteurs de la société grenobloise. Face à la catastrophe ou lors de la cicatrisation des plaies, tous les enjeux internes d'une société « en réparation » s'affirment. L'inondation catalyse de nouveaux rapports sociaux, dominés par des dynamiques de cohésion ou de tensions qui s'entrecroisent. II s'agit, dans ce premier temps de la résilience, d'absorber au mieux le choc de la catastrophe.

\section{I Limiter les impacts de l'inondation : les priorités des acteurs}

Les priorités des acteurs sociaux se cristallisent dans un temps très court, puisqu'il est impossible d'anticiper un tel phénomène au XVIII ${ }^{\mathrm{e}}$ siècle. Seule une certaine rumeur précède la montée de l'eau : lors de chaque inondation "Hors les murs et en ville, mille cris retentissent, ॥(Blanc, I733, v. 90). Face à l'eau qui monte, les stratégies adoptées par les Grenoblois sont variées et se révèlent plus ou moins payantes après la décrue. Si pour un particulier, le sauvetage de l'essentiel de ses biens est faisable, il devient impossible pour un marchand ou un commerçant: «plusieurs marchands ont été ruinés, notamment les épiciers qui ont été surpris par l'eau, sans qu'ils aient eu le temps de pouvoir faire leurs drogues, huiles, sucreries et autres marchandises qui craignant la mouilleure ${ }^{26}$. Ainsi les arbitrages de chacun s'orientent avant tout vers la préservation des biens individuels, afin de mieux pouvoir reprendre une vie normale après l'inondation.

${ }^{26} \mathrm{AD}$ Isère : 3E I 108/7 : Récit de J.-F. Marchand le Jeune
Alors que le siège imposé par l'eau devient imminent, les préoccupations matérielles passent au second plan et la priorité des citadins devient leur propre survie. Cette mise en lieu sûr peut apparaître comme un sauve-qui-peut général; néanmoins certaines logiques sociales sous-tendent ce chaos apparent. La solidarité de voisinage semble privilégiée : «Ceux qui étaient au rez-de-chaussée se logeaient en étages. » (Blanc, I733, v. 270). Cela pose avec acuité le problème de la subsistance pour qui n'a pas, « par précaution, penser à prendre de l'eau, de la chaire, du vin et du pain pour manger. " (d'après Reinier, I74I, orthographe corrigée). L'expérience de 1733 paraît jouer un rôle dans les réactions des citadins de I740, qui cherchent refuge plus promptement, sans pour autant le faire plus sereinement.

Pour les habitants, conscients de la vulnérabilité de leur habitation, la survie passe par la fuite vers un lieu sûr vers les lieux des plus hauts de la ville. II s'agit de choisir entre deux peurs, qui peuvent aujourd'hui s'interpréter en terme de prise de risque : «la peur d'être noyés ou d'être écrasés ॥ (Blanc, 1733, v. 270). Dans cette fuite, la solidarité familiale s'exprime envers les plus vulnérables, femmes, enfants et vieillards.

Pour les élites de la cité, il s'agit de se mobiliser pour faire preuve de la présence et de la bienfaisance, attendues de ceux de leur rang social, en portant secours aux victimes isolées dans leurs refuges ${ }^{27}$. Certaines priorités s'expriment lors de ces secours. Alors que les notables de Grenoble apportent leurs bienfaits aux citadins, les habitants des faubourgs, inondés de toute part et isolés à l'extérieur des remparts, ne voient pas l'ombre d'un secours venir de la ville inondée. Cette forte inégalité cristallisera les tensions au cœur de la résilience.

Durant la catastrophe, la société crée donc les conditions d'une résilience qui débute bien avant l'évacuation des dernières boues. Les infrastructures sont fragilisées, mais le tissu social grenoblois ne se trouve pas déstructuré. La population est indemne et est demeurée dans la ville. Elle n'en reste pas moins bousculée par l'ampleur des pertes matérielles qu'elle n'a pu atténuer que faiblement.

\subsection{Les urgences du lendemain : mendicité et difficulté financière}

Dans la paroisse de Saint-Joseph, la plus sinistrée de Grenoble, seuls $4 \%$ des foyers sont carrément

\footnotetext{
${ }^{27}$ Ce secours se matérialise notamment par la distribution de pain aux sinistrés nécessiteux.
} 
« réduits à la misère ${ }^{28}, 7.5 \%$ des paroissiens sont dits « ruinés », mais pas moins de $28 \%$ sont encore en difficulté quelques semaines après l'inondation. Cette forte proportion de familles pauvres, ruinées ou misérables doit compter sur la charité du reste de la société sinistrée pour survivre durant le temps de résilience.

Au-delà de la mendicité, les difficultés financières des Grenoblois s'expriment une quinzaine de jours après la catastrophe à travers un important pic de contraction d'obligations ${ }^{29}$. Celui-ci se traduit par une accélération de la fréquence de contractions, mais également par un doublement global des montants empruntés. Du point de vue social, le nombre d'enfants abandonnés et recueillis par l'Hôpital général de la ville est en vive augmentation ${ }^{30}$. Mais le phénomène de ruine totale des Grenoblois tient davantage de l'exception et ne doit pas être généralisé.

Si les cadres sociaux sont, dans une certaine mesure, bouleversés par ces quelques ruines individuelles, ce traumatisme reste limité : il est donc plus facilement absorbable par l'ensemble de la société.

\subsection{Les solidarités et leurs enjeux : de la mobilisation des édiles à l'institutionnalisation de la solidarité urbaine}

La société grenobloise semble faire preuve d'une réelle cohésion, stimulée par la catastrophe. L'élan de solidarité parait général sous la plume d'André Blancla-Goutte en 1733 : "Chacun selon sa situation, en ce temps de misère, / Avec ses petits moyens, fit des largesses pour les pauvres. / L'un donna dix louis d'or, l'autre cinq, un autre vingt écus; / Celui-ci son nécessaire, l'autre son superflu. » (Blanc, I733, v. 52 I - 524). Des logiques de don contre don ou de don gratuit recoupent pleinement les liens de parenté, de clientèle ou de voisinage. "Tout ce bien collecté et sagement réparti I Apporta du soulagement à beaucoup de malheureux. ॥ (Blanc, 1733, v. 527 - 528) Une solidarité verticale entre les catégories sociales est activée au sein de la société grenobloise. Elle s'exprime par deux biais : le don philanthropique de pain ou d'argent pour pallier à une situation critique et le dédommagement ou le

\footnotetext{
${ }^{28} \mathrm{AD}$ Isère $2 \mathrm{C} 816, \mathrm{f}^{\circ} \mathrm{I}$

${ }^{29}$ L'obligation est un contrat d'emprunt financier gagé. (AD Isère : 8C II 40, II73 et II74: Registres de contrôle des actes notariés).

${ }^{30} \mathrm{AD}$ Isère : $\mathrm{H}_{+}$GRE/E_I0, $\mathrm{f}^{\circ} 217$
}

dégrèvement $^{31}$ de pertes effectives causées par l'inondation.

Une partie non négligeable de ces dons, en argent ou en nature, sont effectués directement par les notables locaux en leur nom propre (Intendant, Evêque, Prélats, Présidents, etc.) et par les institutions officielles de la ville, où ils sont influents (Favier, 2007). Il semble ainsi que la microsociété que forme l'élite politique grenobloise soit à l'origine de la majeure partie des dons et à l'initiative de nombreuses autres actions de charité. L'action de ces «hommes providentiels ordinaires » est largement diffusée par les récits et survalorisée dans les rapports. Sur le théâtre de la catastrophe, cette surenchère de générosité prend les allures d'une compétition entre les membres de l'élite politique locale.

L'Hôpital général de la ville de Grenoble G2 $^{32}$ constitue l'instrument de la solidarité urbaine. Pourtant l'inondation inhibe fortement l'activité de l'Hôpital. La solidarité d'urgence s'exerce en dehors de ce cadre, puisqu'elle est assurée personnellement par les notables urbains. Dans les semaines qui suivent chaque événement, cette structure d'assistance prend le relais des solidarités individuelles. La mobilisation de l'Hôpital est donc incontestablement plus énergique suite à l'inondation de décembre 1740 que suite à celle de 1733. Pourtant, cet élan s'essouffle largement en février et mars $174 \mathrm{I}^{33}$. En effet, l'Hôpital est trop largement sollicité, alors qu'il ne bénéficie pas de dons extraordinaires. II ne doit compter que sur le revenu de ses terres, alors largement dégradées par l'inondation. Ce problème de financement, inhibant ses activités au moment critique, n'est résolu que par la vente de domaines, l'emprunt et la quête publique

La catastrophe active une entraide familiale et renforce les liens solidaires entre Grenoblois, transcendant les clivages sociaux ${ }^{34}$. Ce resserrement des liens de solidarité à l'intérieur de la cité freine la recherche de fonds à l'extérieur de la ville. De plus Grenoble conserve sa fonction créancière vis-à-vis de son territoire. Les liens internes à la vallée de l'Isère sont eux aussi renforcés. Ainsi une véritable solidarité

\footnotetext{
${ }^{31}$ La pratique du dégrèvement conduit à une diminution de l'impôt dû par le sinistré, cette diminution est proportionnelle aux dommages subis.

${ }^{32}$ Cette structure est en cours d'institutionnalisation au début du XVIIlième siècle.

${ }^{33} \mathrm{AD}$ Isère : $\mathrm{H}_{+}$GRE/E_I 0 et 12

${ }^{34}$ Ces liens spécifiques de solidarité, moins désintéressée, peuvent être mis à jour à la lumière des obligations contractées suite à l'inondation de I740 (AD Isère : 8C II73 et II74).
} 
au sein de la zone sinistrée apparaît spécifiquement durant ce temps de résilience ${ }^{35}$.

Cette solidarité a pourtant ses limites. La cohésion entre les citadins, qui s'affiche à travers l'évacuation des boues, n'est pas si complète qu'il n'y paraît dans les récits. Au lendemain de l'inondation de 1740, il est en effet nécessaire de faire publier deux ordonnances obligeant une entraide générale pour le nettoyage urgent de la ville ${ }^{36}$.

La solidarité qui s'exprime suite aux inondations n'a pas non plus un caractère entièrement spontané. Certains «appels au don » sont lancés dans les rapports officiels en faveur des fermiers sinistrés de la plaine de Grenoble, dont le dédommagement n'est pas à la hauteur des pertes subies. Pour appuyer cette sollicitation de solidarité, il est nécessaire de rappeler à la société grenobloise que «... tout ces malheurs reflueront aussi sur les habitants de la Ville qui ne recevront plus les même secours et provisions que les habitant des granges leurs fournissent si abondamment ${ }^{37}$. Ce type de discours est propre à susciter la solidarité et l'entraide par sa référence à des valeurs communes (Pfister, 2002).

Cette solidarité et cette assistance aux victimes sont essentielles dans le processus de résilience du macrocosme grenoblois. Elle évite la non résilience de la frange la plus vulnérable du système social de Grenoble. Par un certain partage de richesse entre les rescapés de l'inondation, elle apaise les tensions entre les quelques bénéficiaires de la catastrophe et les différentes ruines occasionnées. Nous l'avons vu, cet élan de solidarité provient en grande partie des Grenoblois eux-mêmes sous l'impulsion de leurs élites : la société grenobloise se résilie donc en grande partie par elle-même.

Cette première étape de la résilience sociale a engagé la société grenobloise sur la voie du retour à la normale. Une longue phase de normalisation est alors activée. Cet approfondissement de la résilience reste pourtant peu accessible par le biais des sources ordinaires, qui insistent davantage sur les malheurs des sinistrés que sur le retour progressif à la normale. Nous nous focaliserons donc, lors de l'étude de cette période de retour à l'habituel, sur la conscience accrue des risques qu'elle véhicule. Cette prise de conscience

\footnotetext{
${ }^{35}$ L'aide royale de 15000 livres accordée à la Ville de Grenoble en janvier 1741 constitue néanmoins un fait notable.

${ }^{36}$ AM Grenoble : DD 175

${ }^{37}$ AD Isère : $2 C 816, f^{\circ} I$ : «Observation sur le dénombrement des habitants... »
}

stimule certains réajustements du rapport de la société au risque, des réajustements qui affectent le fonctionnement social.

\section{LES APPROFONDISSEMENTS DE LA RÉSILIENCE : CONSCIENCE, CONSTRUCTION ET INTÉGRATION DU RISQUE DANS LE FONCTIONNEMENT SOCIAL}

Aux $X V I^{e}$ et $X V I I{ }^{e}$ siècles, " une nouvelle interprétation de la catastrophe se met progressivement en place » (Quenet, 2005). Cette mutation participe à la " construction du risque » par les sociétés humaines développée par Grégory Quénet. Nous privilégierons, dans cette étude, le prisme de la «conscience du risque ». En effet, la « conscience du risque » combine de façon active la perception de l'environnement, dans sa dimension risquée, et la réponse apportée à ces stimuli par les sociétés concernées.

\section{I L'inondation passe, le risque augmente, les acteurs s'adaptent}

1732, 1733, 1737, 1739, 1740,... Les crues susceptibles de causer des inondations se multiplient autour de Grenoble, en moins d'une décennie. Dans les consciences, l'inondation passe d'un statut d'événement extraordinaire à celui de catastrophe récurrente. En I778, l'ingénieur Marmillot traduisait la psychose des Grenoblois face à l'augmentation du risque d'inondation au cours du $\mathrm{XVIII}$ e siècle: Grenoble " a souvent éprouvée du passé des moments terribles qui laissent sans cesse de cruelles inquiétudes sur ce qu'elle $a$ à craindre pour l'avenir $)^{38}$.

Une fois le risque douloureusement rappelé par chaque inondation, une réelle psychose s'empare d'une partie de la société à la moindre alerte. En effet, l'expérience d'un accident renforce la conscience de sa potentialité d'occurrence. Cette conjoncture, dans laquelle la catastrophe « devient habituelle», exacerbe alors la prise en compte de ce risque à une échéance variable.

Dans les baux à ferme passés par les monastères grenoblois, la prise en compte du risque d'inondation est temporairement majorée au cours des années qui suivent les deux inondations principales (de 1734 à 1736, ainsi que de $|74|$ à 1743). De façon concomitante, les autres risques climatiques sont davantage pris en considération à travers les contrats.

${ }^{38} A D$ Isère : $J 523, f^{\circ} 13$ 
Paradoxalement, c'est au cœur de la série d'inondations, en 1736, que le risque d'inondation devient perd sa prépondérance parmi l'ensemble des risques intégrés par le milieu agricole. II y a donc clairement une globalisation de l'ensemble des risques et une meilleure prise en compte de ceux-ci par le monde agricole suite aux inondations. L'origine de cette prise en compte du risque par la société du $X V I^{e}$ siècle tient à la fois de la tradition et de l'expérience acquise après chaque épisode catastrophique. Bien plus qu'une simple secousse, l'inondation agit comme une piqûre de rappel de la vulnérabilité globale de la ville de Grenoble et de son territoire. Du haut de son clocher le poète André Blanc-la-Goutte se lamente : « Il fut mal avisé celui qui te construisit si bas! » (Blanc, I733, v. 280). Avec l'augmentation de la fréquence des inondations, les Grenoblois des années 1730 et 1740 ont le sentiment d'habiter une ville très vulnérable.

Cette conscience nouvelle du risque chez les acteurs sociaux influe clairement sur leur propension à prendre des risques. En outre, celle-ci conditionne une large part des conséquences de la catastrophe suivante. Ainsi, le 17 mars 1731, lorsque François Blanchet, modeste cordonnier, loue une chambre basse dans la rue Très-Cloîtres, particulièrement vulnérable aux inondations, il s'engage à verser annuellement 35 livres pendant cinq ans ${ }^{39}$. Fortement touché par l'inondation de 1733, notre cordonnier passe donc automatiquement de la ruine personnelle au surendettement. Ainsi le fait de prendre des trop grands risques conduit à annihiler les capacités d'adaptation de certains Grenoblois.

La propension à prendre des risques est clairement influencée par les inondations. Dans le territoire péri urbain, une certaine recomposition géographique de la prise de risque s'effectue après chaque catastrophe. Chaque risque pris a une forte incidence sur la vulnérabilité de chacun des fermiers. L'influence d'une telle prise de risque sur l'échec ou la réussite de l'exploitation des fermes concomitantes de la catastrophe n'est pas systématique. Néanmoins, certaines tendances se dégagent. Tous les contrats stipulant une prise de risque partagée entre le bailleur et son fermier ${ }^{40}$ se sont traduits par des continuations (prolongement du contrat du fermier sur un même fond). À l'inverse la moitié des contrats dont le risque

\footnotetext{
${ }^{39} \mathrm{AD}$ Isère : $4 \mathrm{G} 56, \mathrm{n}^{\circ} 35$

${ }^{40}$ Le partage de la prise de risque se matérialise par l'indemnisation au fermier du déficit de récolte ou bien par l'annulation du payement de la ferme et le partage des fruits de la récolte endommagée.
}

est entièrement assumé par le fermier ${ }^{4 l}$ a conduit à des départissions (démission du fermier avant le terme de son contrat de ferme). Après les inondations, il y a beaucoup de non renouvellements et de départissions de fermes. Le taux d'échec des fermes est divisé par trois après l'inondation de 1740:80\% des fermes ne font pas l'objet de départissions. Alors que les inondations de la décennie 1730 ont été à l'origine d'un grand renouvellement des fermiers sur les terres des Visitandines, seuls les fermiers en place dans la décennie 1740 restent sur les mêmes terres tout le milieu du XVIII ${ }^{e}$ siècle. La catastrophe assure donc une sorte de mainmise sur les terres par les quelques familles de fermiers qui ont su la traverser.

L'accroissement de la conscience du risque ne se limite pas aux risques empiriques (dont la manifestation est connue des Grenoblois). II met spécifiquement en avant d'autres formes de risque qui sont alors survalorisées ou minimisées.

\subsection{De nouveaux risques, entre construction et acceptabilité}

Le risque est une construction sociale, un concept élaboré qui permet à une société de passer d'une perception ponctuelle d'un danger à une prise en compte consciente d'une menace potentielle (Quenet, 2005). Si, au XVIII ${ }^{\text {e }}$ siècle, le risque d'inondations est déjà élaboré depuis longtemps, d'autres risques connexes se développent dans les consciences. Chaque inondation agit tel un catalyseur de réflexions participant à la construction de « nouveaux risques ».

Le risque se complexifie. II est perçu de façon "generalle et préjudiciable » pour Grenoble et ses environs. II est également conçu comme durable. À la suite de la catastrophe de 1740, "le dommage est beaucoup plus considérable qu'on ne saurait le penser; et durera pendant plusieurs années ${ }^{42}$. Le risque hydrologique devient un risque économique général, puisque les inondations " ont rendu plusieurs fois les routes absolument impraticables, ce qui a toujours causé à Grenoble et aux environs une augmentation sur le prix des danrées ». II s'étend également à «tout ce pays [...] ruiné par l'interruption de la communication et du commerce [...] et la privation des secours, et des provisions qu'on tire respectivement d'un côté $a$ l'autre $1{ }^{43}$. Le risque est aussi sanitaire puisque dès le

\footnotetext{
${ }^{41}$ Le contrat est alors passé « aux périls et risques dudit fermier » (AD Isère : $4 \mathrm{G} 64, \mathrm{f}^{\circ}$ |402/4)

${ }^{42} A D$ Isère : $2 C 816, f^{\circ} \mid$

${ }^{43}$ AM Grenoble : DD 175
} 
23 décembre 1740, les boues dans la ville font ressurgir le spectre épidémique. La priorité des autorités devient alors d'« éviter les maladies qu'elles [les boues] pourraient causer ${ }^{44}$. Enfin les autorités prennent des mesures contre le risque d'accidents, puisque «l'inondation a détrempé les fondations des maisons de cette ville, en telle sorte qu'il y aurait du danger de laisser rouler les voitures dans les rues ${ }^{45}$. Au cours de la décennie 1730, la potentialité de catastrophes est largement envisagée. Pour la société grenobloise, la question centrale devient alors celle de l'acceptabilité des risques.

L'acceptabilité d'un risque pour une société est le degré de tolérance qu'elle peut avoir vis-à-vis de la réalisation de ce risque. Les enjeux sociaux et économiques liés au risque, et leur perception par les différents acteurs, modulent le seuil de l'acceptabilité. Même face à la possibilité d'une catastrophe, les victimes potentielles considèrent davantage les dommages immédiats préjudiciables à court terme, que les bénéfices à long terme.

Cependant, un cas intéressant de changement dans l'acceptation du risque est perceptible à travers de multiples ordonnances de l'Intendance. Celles-ci menacent les populations riveraines qui « coupent des saules et peupliers et détruisent lesdites réparations» sur les digues ${ }^{46}$. La protection assurée par les digues est reconnue par tous, mais le besoin en bois de chauffage des foyers urbains semble considéré comme prioritaire par rapport à cette protection. Pour de nombreux habitants, les logiques économiques à court terme et la nécessité immédiate l'emportent sur les logiques de prévention des risques à plus long terme, « ce qui est préjudiciable et pourrait à l'avenir rendre inutiles lesdites réparations $\${ }^{47}$. À la faveur de la série d'inondations qui a eu lieu, l'acceptation du risque d'inondation diminue. L'intérêt des digues semble mieux perçu par les riverains. Les pratiques individuelles nuisibles à la prévention diminuent de façon visible.

Le risque est parfois collectivement minimisé au profit de la conservation de structures vitales pour les Grenoblois (maisons, canaux d'évacuations,...). II s'agit souvent d'économiser de coûteuses dépenses de

\footnotetext{
${ }^{44}$ Ibid.

${ }^{45}$ AM Grenoble : DD 175 (23 décembre 1740). La circulation des carrosses est prohibée en ville puisque les vibrations de leur passage seraient susceptibles de provoquer l'effondrement des immeubles aux fondations fragilisées.

${ }^{46} \mathrm{AD}$ Isère : $2 \mathrm{C} 783$

${ }^{47}$ lbid.
}

protection, tel un projet de canal de dérivation de l'Isère au sud de la ville. Les enjeux extérieurs dépassent alors le risque que les acteurs ne considèrent pas comme primordial à court terme. Certains enjeux supérieurs peuvent mener à des acceptations détournées du risque. Pourtant les enjeux de la conservation de la capitale dauphinoise ne laissent place à aucune prise de risque.

\subsection{Sauver Grenoble des flots}

Pour la plupart des décideurs, dès les mois qui suivent la catastrophe de 1733 et pour tout le XVIII siècle, le risque de submersion de Grenoble est l'un des enjeux majeurs. Le seul maître mot devient alors : «Sauvez Grenoble des flots». La psychose se traduit par l'élaboration de véritables scénarios-catastrophes prédisant les prochaines inondations de Grenoble, dans un futur indéterminé. Ainsi les risques «sont surtout à craindre pour la ville de Grenoble et le territoire qui l'environne [..., la ville] est actuellement exposée $a$ être entièrement détruite du moins dans sa partie la plus précieuse a la première crue des eaux ${ }^{48}$. Ces prédictions, formulées le 15 décembre 1739, sont volontairement alarmistes; en annonçant la fin prochaine de la ville, elles visent à «prévenir Grenoble d'une destruction prochaine et quant à présent inévitable ${ }^{4}{ }^{49}$. Prédire la catastrophe pour générer une mobilisation face au risque et éviter ainsi que le risque ne se réalise, le procédé est commun.

Nous l'avons mentionné les logiques à court terme des communautés riveraines en matière de prévention s'opposent à la mise en place d'une prévention globale. Néanmoins, en matière de prévention locale, les riverains semblent mieux armés pour faire face aux débordements. Force est de constater que, dans les années 1730, la collaboration de ces deux approches n'est que balbutiante.

La raison de cette divergence réside sûrement dans les choix de priorité effectués par les autorités grenobloises en matière de prévention. En 1739, il est rappelé que «l'ingénieur des ponts et chaussées ne doit point perdre de vue l'objet de la conservation de Grenoble $1{ }^{50}$. Les pressions royales et provinciales se conjuguent à celles exercées par les bourgeois grenoblois pour « mettre à couvert la Ville de Grenoble des inondations " ${ }^{51}$ par tous les moyens. La maîtrise des financements de

\footnotetext{
${ }^{48} \mathrm{AD}$ Isère : 2C 784 : «Mémoire sur le danger pressant ou le torrent du Drac met la ville de Grenoble... ».

49 Ibid.

${ }^{50}$ BM Grenoble : R 7214 : « Mémoire sur le torrent du Drac... ».

${ }^{51}$ BM Grenoble : 010021
} 
l'endiguement joue également un rôle important dans ce choix. Lors de l'élévation des digues, qui se réalise d'avantage en amont qu'en aval de la ville, cette priorité se traduit par une attention supérieure portée au « bord qu'on prend soin d'élever et fortifier du côté de Grenoble ${ }^{52}$. Ainsi l'endiguement qui protège les paroisses de la rive gauche du Drac est clairement et volontairement moins résistant que celui mettant à l'abri Grenoble sur la rive opposée (Cœur, 2003).

Le $\mathrm{XVIII}$ e siècle marque une diminution de l'acceptabilité du risque par les sociétés. L'aménagement volontariste des ingénieurs trouve sa justification dans cette conscience nouvelle du risque. Elle s'est particulièrement exacerbée durant le temps d'approfondissement de la résilience qui succède à chaque catastrophe. Ainsi le processus de résilience reconduit la société vers l'essentiel de sa structure initiale. Néanmoins, il catalyse une intégration plus complète du risque dans son fonctionnement social. Les apports de cette résilience en matière de risque constituent une impulsion réelle pour la construction sociale du risque. Ce rapport nouveau au risque d'inondation trouve ces racines en partie dans une longue tradition de gestion du territoire vulnérable. Ces temps de reconstruction sociale de "l'aprèscatastrophe » stimulent également l'expression d'une culture locale du risque sur le territoire grenoblois.

\section{UNE GESTION DURABLE DES ESPACES PÉRI-URBAINS À RISQUE : UNE AUTRE DIMENSION DE LA RÉSILIENCE}

La définition de la culture locale du risque reste soumise à débat. Dans une acception large, il s'agirait d'un ensemble de phénomènes matériels et idéologiques, polarisés autour d'un risque, caractéristiques d'un groupe d'individus. En effet, la polarisation de la culture du risque est conditionnée par la conscience inhérente du risque. Pourtant, elle est loin de se limiter à la conscience au quotidien de la possibilité de catastrophe. Elle couvre également un ensemble de pratiques héritées et pérennisées. Ces pratiques relèvent de la tradition, mais sont aussi modelées au gré des expériences catastrophiques. Parmi les multiples facettes de cette culture du risque, figure l'entretien de l'espace agricole périurbain exposé aux crues de l'Isère.

Ces pratiques traditionnelles se construisent et se sédimentent dans le paysage. Elles sont accompagnées de règles tacites, ou rappelées régulièrement, qui agissent comme de véritables garants de la

\footnotetext{
${ }^{52}$ BM Grenoble : R 7215 : « Mémoire sur le torrent du Drac... ».
}

préservation de l'espace face au risque d'inondation. La terre constitue un des biens les plus précieux au $\mathrm{XVIII}$ siècle. Elle garantit d'importants revenus aux institutions et aux notabilités urbaines propriétaires de ces fonds. Le bon entretien de la terre et de sa fertilité apparaît donc comme une dimension essentielle de la préservation des revenus des urbains face au risque d'inondation. Cette gestion durable de l'espace agricole soumis au risque d'inondation passe par la conservation des boisés et des fossés dans la plaine inondable de Grenoble, deux pratiques institutionnalisées.

\section{I Les arbres en zone inondable : enjeux, gestions et mutation}

«Des milliers d'arbres tristes débris de ces riches héritages sont entraînés par le torrent $\nu^{53}$, lors de chaque inondation. Le bois constitue une denrée de valeur au $\mathrm{XVIII}{ }^{\mathrm{e}}$ siècle. Ses nombreux usages et la fin des possibilités de défrichement ont conduit à créer une véritable situation de pénurie en Dauphiné ${ }^{54}$. Ainsi, la plantation d'arbres sur les digues fait l'objet d'une extrême attention. L'inspecteur des Ponts et Chaussées, M. Fayolle, détaille les usages et avantages de cette opération ${ }^{55}$ :

\begin{abstract}
on tirera de cette plantation soit pour la solidité que cela donnera au terrain contigu aux digues soit par le produit des ventes ou par l'employ qui sera fait de ce même bois [...] pour la construction des nouvelles digues qui couteront la moitié moins attendu qu'on trouvera le bois sur place.
\end{abstract}

En effet, grâce à la gestion durable du stock de bois qu'il projette, il réussit à rendre rentable son projet d'endiguement.

Le bois est donc un enjeu économique majeur, mais il a également une importante vertu écologique. Ainsi, « on plantera des plançons de peuplier qui rendront ces atterrissements estables » en janvier $1741^{56}$. Les espèces végétales sont ainsi sélectionnées pour leurs vertus stabilisatrices du sol et pour leur capacité à croître en milieu humide. «De petits ormes et fresnes

\footnotetext{
${ }^{53} A D$ Isère : $J 523, f^{\circ} 13$

${ }^{54}$ Selon les constatations de l'ingénieur Pierre Roland en I74I (AD Isère : $\left.2 \mathrm{C} 816, \mathrm{f}^{\circ} 9\right)$.

${ }^{55} \mathrm{AD}$ Isère : $2 C 783$

${ }^{56} A D$ Isère : $2 C 816, f^{\circ} 6 b$ is
} 
dont les racines consolideront le terrain $)^{57}$ sont préconisés sur les rives. Dans la plaine de Grenoble, le paysage classique reste le «pré et terre entourrée de saules et peupliers ${ }^{58}{ }^{5}$. Cette végétation permet non seulement de stabiliser et de drainer le sol, mais aussi de jouer un rôle actif durant l'inondation en permettant «que les graviers que ce torrent roule et entraîne lors de ses irruptions fussent arrêté par ce bois et plantement et même les eaux des irruptions divisées $)^{59}$.

Le double enjeu, économique et écologique, des arbres dans la plaine de Grenoble est une forte source de tension entre les différents acteurs de ce territoire. Les fermiers n'ont pas d'intérêts économiques sur le fond au-delà de la durée de leur ferme. De leur côté, les propriétaires de la terre envisagent une gestion beaucoup plus durable pour la conservation de leurs biens. Ainsi, les monastères urbains se réservent largement l'exclusivité de l'exploitation de ces arbres. De cette façon, la communauté religieuse s'assure un approvisionnement en bois régulier et autonome, ainsi qu'une protection optimale des terres qu'elle possède. Par ce biais, la densité d'arbres bordant les champs semble s'accroître dans cet espace à risque, puisque très souvent, " ledit fermier promet et s'oblige de planter des saules et peupliers autour du pré ${ }^{60}$, « aux endroits les plus nécessaires ${ }^{61}$.

Pour arbitrer entre ces intérêts divergents, les contrats de fermes mentionnent des modalités strictes de gestion des arbres. La gestion rigoureuse du stock d'arbres est une pratique traditionnelle. II s'agit d'une gestion à long terme, qui n'est pas initiée par la catastrophe, mais qui paraît réactivée avec vigueur dans le temps de la résilience. Ainsi, les baux à ferme du pré Pichat, proche des Granges au sud de Grenoble, mentionnent déjà en 1680 l'obligation du fermier qui « plantera annuellement trois douzaines de plançons qu'il convinira $)^{62} .86$ ans plus tard, le fermier de ce fonds doit toujours planter « chaque année au tour du fond cinquante plaçons saules et peupliers aux les endroits les plus convenables $\|^{63}$. À l'inverse, en octobre 1733, « en considération du dommage que l'innondant et la Rivière d'lzere lui a fait dans ladite ferme, promet aussi ledit mollard de planter tous les plançons nécessaires audit domaine ainsi

\footnotetext{
${ }^{57}$ Ibid.

${ }^{58} A D$ Isère : 4G 64, $f^{\circ}$ I402/5 (29 juin I74I).

${ }^{59} A D$ Isère : $2 C 783$

$60 \mathrm{AD}$ Isère : $4 \mathrm{G} 64, \mathrm{f}^{\circ}$ 1402/3

$61 \mathrm{AD}$ Isère : $3 E \mathrm{E}$ 1435/14, $\mathrm{f}^{\circ}$ 184 - 185

62 AD Isère : $7 \mathrm{H} 75$

${ }^{63}$ Ibid.
}

qu'il est prévu par ledit contrat ${ }^{64}$. II convient alors de s'intéresser aux permanences et aux éventuelles mutations initiées par les inondations.

Certaines dispositions des baux à ferme incitent à une gestion durable des saules et des peupliers ${ }^{65}$, alors que d'autres contrats permettent la destruction des boisés présents autour des fonds. En tant que remède aux dégradations de l'inondation, l'interdiction de coupe devient systématique pendant deux ans après l'inondation de $1733^{66}$. L'obligation de plantation nouvelle est bien stimulée par l'événement catastrophique. II faut en effet, remplacer les arbres emportés et stabiliser et drainer la terre inondée. La fragilité des terres labourées est particulièrement prise en compte entre 1736 et 1742 . Durant cette période, $83 \%$ des baux à ferme contractés pour des terres de labour mentionnent cette obligation de plantation. II apparaît clairement que l'inondation de 1733 met fin aux pratiques destructrices d'exploitation de ces arbres. La dernière autorisation d'exploitation du bois présent sur la ferme date d'avril 1733. La gestion des arbres est donc relativement corrélée à la conjoncture catastrophique. D'une part, l'inondation aurait mis en évidence le caractère néfaste pour la conservation du fond agricole de certaines pratiques intensives d'exploitation du bois. D'autre part, la plantation permet de cicatriser les plaies ouvertes par l'inondation sur les terres agricoles.

Au début du $X V I I I^{e}$ siècle, certaines pratiques agricoles de modération du risque sont présentes dans la plaine de Grenoble. Mais les années 1733 et 1740, avec leurs inondations, réactivent la protection des terres par la préservation des arbres qui bordent les parcelles. Cette sélection des pratiques agricoles par la catastrophe se combine à la volonté des propriétaires urbains de préserver leurs biens. Cette convergence élabore une réelle gestion durable des arbres dans les parcelles exposées. Elle s'intègre aisément au sein d'une culture locale du risque.

\subsection{Entretenir les fossés, anticiper sur le risque}

Un autre élément de l'entretien du territoire est particulièrement sensible dans le temps de résilience : l'entretien des fossés en prévision d'une prochaine catastrophe. Le rôle fondamental d'amortisseurs de crues joué par les fossés, à Grenoble ou dans la

\footnotetext{
${ }^{64} A D$ Isère : $3 E$ 1435/14, $f^{\circ} 194-195$

${ }^{65}$ La gestion durable des boisés se caractérise par exemple par l'interdiction de coupe ou par l'obligation de plantation nouvelle.

${ }^{66}$ Pourtant de façon étrange l'inverse est constatable après celle de 1740.
} 
campagne environnante, est mis en évidence à chaque inondation.

Si les fossés de la ville n'avoient été encombrés et presque pleins de boue et de plus traversés par des ponts et leur conduite $[\ldots]$ une grand partie des eaux aurait pu s'écouler par là, ce qui aurait beaucoup diminué la grandeur de l'inondation. ${ }^{67}$

Dans Grenoble, les enjeux qui entourent les ruisseaux qui traversent la ville sont d'ordre économique et sanitaire. Si l'écoulement y est bon, ces petits cours d'eau permettent de faire fonctionner les moulins, comme celui de Très-Cloîtres ${ }^{68}$. Ils jouent également le rôle d'égout collecteur des déchets de la ville vers l'Isère. Ainsi en ville, les fossés sont vitaux, mais leur bon écoulement est régulièrement entravé, gênant l'évacuation des eaux lors de la décrue. En temps normal, « il est d'usage que [...] le grand repurgement de ce canal se fait tout les trois ans a la
En zone rurale la problématique est différente. II est obligatoire pour les fermiers « de tenir ledit fossé repurgé et en état d'y recevoir les eaux ${ }^{70}$, afin de pouvoir drainer correctement les terres avoisinantes et de prévenir le dépôt de boues et l'érosion en cas d'inondation ou de forte pluie. L'obligation de curage des fossés par le fermier semble faire partie des pratiques habituelles et des préoccupations majeures de gestion de l'espace rural. II s'agit de préserver le patrimoine agricole en cas d'inondation ${ }^{71}$ et les infrastructures vitales du royaume ${ }^{72}$.

Les contrats établis durant le temps de la résilience insistent tout particulièrement sur cette pratique. Lorsque Jean Maillet, meunier aux Granges, contracte un bail à ferme le 27 septembre 1733, un article entier est consacré à ses obligations d'entretien post-catastrophique : "sera soigneux lors des crues et abondance des eaux de tenir le ruisseau vers la prise d'eau bien purgé pour que lors des abondances elle aye son cours dans icelluy pour prévenir les inondations qui

Tableau I

Éléments d'adaptation conduisant à la résilience de la société grenobloise suite aux inondations de 1733 et 1740

\begin{tabular}{|c|c|c|}
\hline \multicolumn{2}{|r|}{ En ville } & En milieu péri-urbain \\
\hline \multirow{2}{*}{$\begin{array}{l}\text { Durant l'inondation, } \\
\text { phase préliminaire à la } \\
\text { résilience }\end{array}$} & \multicolumn{2}{|c|}{$\begin{array}{l}\text { - Limiter ses pertes matérielles, mettre en sécurité ses biens mobiliers } \\
\text { - Se mettre en sécurité } \\
\text { - Assurer ses besoins alimentaires }\end{array}$} \\
\hline & $\begin{array}{l}\text { - Générosité des élites } \\
\text { - Assistance aux sinistrés nécessiteux } \\
\text { - Solidarité de voisinage } \\
\text { - Sécurisation de la ville face au pillage }\end{array}$ & - Isolement des foyers \\
\hline \multirow[t]{2}{*}{$\begin{array}{l}\text { Lors de la phase } \\
\text { d'urgence de la } \\
\text { résilience }\end{array}$} & \multicolumn{2}{|c|}{$\begin{array}{l}\text { - Fonctionnement d'une solidarité de voisinage, de parenté ou de clientèle } \\
\text { - Organiser la charité publique par l'élite urbaine et l'Hôpital général } \\
\text { - Survivre et tenter de palier à ses pertes par l'endettement } \\
\text { - Organisation de l'approvisionnement urbain }\end{array}$} \\
\hline & $\begin{array}{l}\text { - Développement des liens financiers internes à la ville } \\
\text { - Nécessité d'appel à l'entraide collective pour l'évacuation des } \\
\text { boues }\end{array}$ & $\begin{array}{l}\text { - Augmentation de la dépendance financière à } \\
\text { l'égard des citadins } \\
\text { - Insistance forte pour que la ville prenne en } \\
\text { considération les pertes des fermiers péri- } \\
\text { urbains }\end{array}$ \\
\hline \multirow[t]{2}{*}{$\begin{array}{l}\text { Lors de la phase } \\
\text { d'approfondissement } \\
\text { de la résilience }\end{array}$} & \multicolumn{2}{|c|}{$\begin{array}{l}\text { - Difficultés économiques et sociales au redémarrage issues des conséquences de la catastrophe, puis } \\
\text { reprise progressive des activités quotidiennes et stabilisation économique } \\
\text { - Prise de recul avec la catastrophe qui conduit à l'augmentation de la conscience du risque. Cela } \\
\text { participe à la construction du risque et à l'intégration de ce risque dans le fonctionnement social } \\
\text { - Diminution de l'acceptation des risques }\end{array}$} \\
\hline & $\begin{array}{l}\text { - Meilleure prise en compte des risques économiques, } \\
\text { sanitaires, etc. qui confèrent une portée plus longue et plus } \\
\text { globale à la catastrophe } \\
\text { - Elaboration de scénarios catastrophes } \\
\text { - Promouvoir de nouvelles mesures de protection de la ville } \\
\text { mais réticence à en assumer le financement } \\
\text { - Appel des habitants aux autorités pour les protéger du risque }\end{array}$ & $\begin{array}{l}\text { - Meilleur partage de la prise de risque } \\
\text { agricole entre les différents acteurs } \\
\text { - Gestion locale du risque } \\
\text { - Amélioration de l'entretien et de l'aménagement } \\
\text { durable de leur territoire face au risque }\end{array}$ \\
\hline
\end{tabular}

charge des différents propriétaires $1{ }^{69}$.

\footnotetext{
${ }^{67} A D$ Isère : $2 C 816, f^{\circ} 10(25$ janvier 174 I)

${ }^{68}$ AM Grenoble : 6Fi 42

${ }^{69}$ AM Grenoble : 3 O 15
}

\footnotetext{
${ }^{70} \mathrm{AD}$ Isère : $2 \mathrm{C} \mathrm{II}$

${ }^{71}$ Parmi les baux à ferme, $60 \%$ le stipule (AD Isère : $23 \mathrm{H} 76$ ), mais la pratique dépasse les obligations contractuelles.

${ }^{72}$ Le 22 juillet 1735, l'Intendance intime l'ordre à des riverains de la route Lyon - Grenoble de faire réparer à leurs frais la route endommagée à cause du mauvais écoulement des fossés de leur communauté ( $A D$ Isère : $2 C \mathrm{II})$.
} 
pourraient nuire aux bâtiments $)^{73}$. Pour les fermiers, la fréquence de cette obligation est supérieure à la normale entre 1736 et $1742^{74}$. Le besoin de nettoyage des fossés et de drainage des sols se combine avec la conscience de l'intérêt de cette pratique pour la conservation des terres arables en situation de risque ${ }^{75}$. Pourtant, ces mentions semblent s'estomper largement à partir de 1742. La conscience accrue du besoin de prévention, déjà mis en évidence, se relâche donc rapidement une fois les effets de la catastrophe gommés.

Gérer l'espace agricole de la plaine de Grenoble de façon durable face au risque, c'est avant tout garantir la pérennité de la valeur des terres agricoles et assurer la conservation du bien affermé, tout en évitant de faire baisser sa productivité. II faut donc concilier les intérêts à court terme des fermiers et ceux à plus long terme des propriétaires. Le risque se superpose alors à tous ces enjeux, faisant planer une menace similaire sur le fermier des faubourgs et son bailleur urbain. Ce risque nécessite une attention commune passant par le biais d'une série de pratiques traditionnelles et remodelées au gré des expériences d'inondations et des orientations de la résilience. Ces pratiques d'entretien régulier de l'espace agricole pour en réduire la vulnérabilité en cas d'inondation constituent un versant important de la culture du risque. II s'agit d'une lutte traditionnelle contre le risque d'inondation dénotant une réelle adaptabilité des sociétés face aux variations du risque réel ou conscient.

\section{CONCLUSION}

Les deux inondations de 1733 et 1740 ont été durement ressenties par la société de l'époque et marquent l'histoire de la ville comme des catastrophes majeures. Néanmoins, leur impact et la résilience dont le système social grenoblois a su faire preuve n'ont pas été de nature à bouleverser durablement une société stable d'Ancien Régime. Si la capacité d'adaptation de quelques rares individus en situation de précarité ou de faible résistance aux changements a été dépassée, ces cas restent minoritaires. La cohésion de cette société urbaine et l'impulsion donnée par ses élites paraissent avoir permis d'adoucir et de digérer les conséquences de ces problèmes d'adaptation. Elles semblent aussi avoir aidé le système socio-économique grenoblois à perdurer au-delà de chaque catastrophe.

\footnotetext{
${ }^{73} A D$ Isère : $3 E ~ I I 8 I / 7, f^{\circ} 381$

${ }^{74} \mathrm{AD}$ Isère : $23 \mathrm{H} 76$

${ }^{75}$ Les terres arables représentent $76 \%$ des obligations de nettoyage des fossés (lbid.)
}

Le prisme du processus de résilience permet d'appréhender les conséquences de la catastrophe audelà des seules destructions. Des dynamiques de cohésion et de tension se conjuguent au sein de cette société chahutée par les eaux. Prises de risque, priorités individuelles et solidarités socio-économiques font paraître l'inondation et sa résilience comme des catalyseurs de mutations sociales mineures. Le processus de résilience de la société et les éléments d'adaptation mis en œuvre par ses membres se positionnent avec leurs propres rythmes (voir Tableau I). Dans les premiers temps du processus de résilience, les arbitrages des acteurs sociaux catalysent la capacité de survie du système grenoblois au-delà de la catastrophe. La seconde phase d'approfondissement comporte deux caractéristiques principales: une normalisation progressive de la vie quotidienne et une tendance à la reproduction du système grenoblois. Cela n'exclut pas pour autant certaines adaptations allant dans le sens d'une plus grande prise en compte des risques.

La résilience présente de multiples facettes: sociale, économique, environnementale, etc. Or les conséquences de l'inondation placent la société face aux problématiques de gestion d'un territoire exposé. II apparaît que la résilience et l'adaptation économicoenvironnementale de ce territoire se fait dans le sens d'une plus grande prise en compte des risques. Cette conscience réactivée participe à la construction du risque dans les sociétés modernes. L'inondation oriente donc les acteurs vers une gestion plus durable de ce territoire à risques.

S'intéresser à la notion de résilience au sein de l'histoire sociale des risques permet de centrer la réflexion sur les actions humaines et sociales à la fois face aux conséquences de la catastrophe et face au risque. Cette approche ne véhicule pas un nouveau paradigme, mais elle revient à ne pas surévaluer la vulnérabilité des sociétés en amont de la catastrophe. Cela permet de dépasser les théories de la passivité des sociétés anciennes face au risque. En sciences sociales, le rapport au risque et la réaction des sociétés anciennes face aux inondations donne la possibilité d'enrichir la problématique actuelle de la gouvernance des risques. Cela apporte également une nuance à l'impression d'accroissement des risques qui nous entourent.

De façon primordiale, la catastrophe est une rupture. Analyser la résilience revient donc à problématiser la portée de la catastrophe en termes de rupture brutale ou de discontinuité d'un système 
métastable ${ }^{76}$. II est possible d'affirmer qu'au regard de l'Histoire, les inondations de 1733 et 1740 se conçoivent comme des catalyseurs marginaux de mutations socio-économiques locales et comme des catalyseurs fondamentaux pour l'approche du risque à Grenoble au XVIII siècle. Une résilience active et prolongée se mute en une prise en main durable de la question du risque par l'ensemble de la société : il s'agit d'une pierre supplémentaire à l'édifice du risque en construction.

\section{BIBLIOGRAPHIE}

BenASSAR, B. (1996). Les catastrophes naturelles dans l'Europe médiévale et moderne, Toulouse, Presses universitaires du Mirail, 269 p.

Berlioz, J. et G. Quenet (2000). « Catastrophes : définition et documentation ", in FAVIER, R. et A.M. GRANETABISSET, Histoire et mémoire des risques naturels, SaintMartin d'Hères, MSH-Alpes, HESOP, pp. 19-37.

Blanc, A. (1733, 1860, 1966). Grenoblo Malhérou, Poésies en Patois du Dauphiné, Grenoble, Imprimerie Dardelet. (Traductions du franco-provençal selon : HOYER, G. et G. TUAILLON (2003). Blanc-La-Goutte, poète de Grenoble (œuvre complètes), Grenoble, Centre alpin et rhodanien.

CYRULNIK, B. (200I). Les vilains petits canards, Paris, Odile Jacob, 24Ip.

CGUR, D. (2003). La maîtrise des inondations dans la plaine de Grenoble (XVIle-XXe siècle) : enjeux techniques, politiques et urbains. Thèse de doctorat d'Histoire et d'Aménagement, Grenoble II, Université Pierre-Mendès, France, 345 p.

FAVIer, R. (1993). Les villes du Dauphiné aux XVIle et XVIIle siècles, Grenoble, PUG, 512 p.

FAVIER, R. (2007). « De la solidarité à l'individualisation des aides. Les dispositifs d'assistance face à l'essor du libéralisme en France au $18 \mathrm{e}$ siècle $》$, in FAVIER, R. et A.M. GRANET-ABISSET, Solidarité et assurance, Les sociétés européennes face aux catastrophes (I7e-2/e siècles), Grenoble, MSH-Alpes, pp. 57-79.

GAILLARD, J.-C. (2007). « Resilience of traditional societies in facing natural hazards », Disaster Prevention and Management, vol. 16, no 4, p. 522-544.

Parde, M. (1928). «Périodicité des grandes inondations et crues exceptionnelles », Revue de Géographie Alpine, vol. 16, p. $499-519$.

Pelling, M. (2003). The Vulnerabilities of Cities: Natural Disasters and Social Resilience, Londres, Earthscan, 256 p.

Pfister, Ch. (2002). Le jour d'après, Surmonter les catastrophes naturelles: le cas de la Suisse entre 1500 et 2000, Berne, Paul Haupt, 263 p.

${ }^{76}$ Passage d'un équilibre à un autre qui ne lui est pas radicalement opposé.
QUENET, G. (2005). Les tremblements de terre aux XVIle et $X V I I l e$ siècles, La naissance d'un risque, Seyssel, Champ Vallon, $587 \mathrm{p}$.

ReINIER, A. (I74I). Grenoblo inonda, ou recit circonstancia du malheurs qu'a causa l'inondation arriva lo vingt-un décembro mil sept cens quaranta. Poemo patois. Grenoble, Imprimerie André Faure.

Walker, B., C.S. Holling, S. Carpenter et A. Kinzig (2004). « Resilience, Adaptability and Transformability in Socialecological Systems », Ecology and Society, vol 9, n० 2, art 5. (www.ecologyandsociety.org/vol9/iss2/art5).

\section{SOURCES}

\section{Archives départementales de l'Isère (ADI) :}

2C II: «Ordonnances de l'intendant Fontanieu » (I724 4I)

2C 783 - 784 : «Aménagements et mémoire sur les débordements du Drac » (1694 - 1756)

2C 816: «Mémoires concernant les moyens de garantir la ville de Grenoble d'une inondation » (I74I)

8C II40, II73 - II74: «Registres de contrôle des actes notariés du bureau de Grenoble » $($ I733, I740 - 4I)

3E I 108/7: « Minutier de M. Marchande le jeune » (I733 34)

3E II8I/7 : « Minutier de M. Revol » (I733 - 34)

3E I435/I4: « Minutier de M. Dusser » (1733)

4G 56 : «Baux à loyer de maisons dépendantes de l'évêché de Grenoble » (1678 - 1760)

4G 64 : « Baux à ferme de l'évêché de Grenoble, Fond des Tailles » $(1727-64)$

7H75: «Biens possédés par les Dominicains de Grenoble aux Granges de Grenoble » (XVIIlième siècle)

23H 76 : «Baux à ferme des Visitandines de Grenoble à St Martin d'Hères » (XVIIlième siècle)

$\mathrm{H}+$ GRE/E_I 0 et 12 : « Livre des délibérations du bureau de la direction de l'hôpital général de la Ville de Grenoble » (I733- 34 et I740-4I)

J 523 : « Mémoires concernant les inondations » (1778)

\section{Archives municipales de Grenoble (AMG) :}

DD 175 : «Mémoires, ordonnances et correspondences relatives aux inondations » $(|730-4|)$

$6 \mathrm{Fi} 42$ : «Affiche pour l'évacuation des boues » (I74I)

3 O 15: "Canaux et ruisseaux, Verderet et Très Cloître " $(1710-1855)$ 
EUE • Les inondations de 1733 et 1740 à Grenoble • a-89

Archives de la bibliothèque municipale de Grenoble (BMG) :

O 10 021: «Requêtes des communautés de Seyssins, Seyssinet, Fontaine, Sassenage suite aux irruptions du Drac » (1740)

R 7214 - 7215: «Mémoires sur le torrent du Drac...» $(1739-40)$

R 8742 : «Témoignage de Pierre Bémond sur l'inondation de 1733 à Grenoble » 